\title{
Morphology, Tensile Strength and Thermal Behavior of Isotactic Polypropylene/Syndiotactic Polystyrene Blends Compatibilized by SEBS Copolymers
}

\author{
Bin Chen, ${ }^{\dagger, \dagger \dagger}$ Tao TANG, Xiliang LI, ${ }^{*}$ Shengqing Xu, Xuequan ZHANG, and Baotong HuAng \\ State Key Laboratory of Polymer Physics and Chemistry, Changchun Institute of Applied Chemistry, Chinese Academy of Sciences, \\ Changchun, Jilin 130022, People's Republic of China \\ ${ }^{*}$ Center of Electron Microscopy, College of Biology, Northeastern Normal University, \\ Changchun, Jilin, 130021, People's Republic of China
}

(Received May 28, 2003; Accepted February 2, 2004)

\begin{abstract}
The effects of three triblock copolymers of poly[styrene- $b$-(ethylene- $c o$-butylene)- $b$-styrene] (SEBS) of different molecular weight (MW) on the morphology, tensile strength and thermal behavior of isotactic polypropylene/syndiotactic polystyrene (iPP/sPS, 80/20) blend are investigated. Morphology observation shows that both the medium MW and the lower MW SEBS are more effective than the higher MW SEBS in compatibilizing the blends. Tensile tests revels both the medium and low MW compatibilizer lead to a significant improvement in tensile strength, while the higher MW compatibilizer is efficient in increasing the elongation at break of the blends. The localization of compatibilizers in the blends is observed by mean of SEM and the correlation between the distribution of the compatibilizers and mechanical properties of the blends is evaluated. The mechanical properties of the iPP/sPS blends depend on not only the interfacial activity of the compatibilizers but also the distribution of the compatibilizer in the blend. Addition of the compatibilizers to the blend causes a remarkable decrease in the magnitude of the crystallization peak of sPS at its usual $T_{\mathrm{c}}$. Vicat softening points demonstrate that the heat resistance of $\mathrm{PP} / \mathrm{sPS}$ blend is much higher than that of the pure iPP.

KEY WORDS Syndiotactic Polystyrene / Isotactic Polypropylene / Polymer Blend / Compatibilization / Morphology /
\end{abstract}

Polymer blending has become an efficient way of developing new materials with tailored properties and improving some deficient performances of many polymers. Isotactic polypropylene (iPP), one of the most important and widely used thermoplastics, excellent in processability, high in stiffness and strength, is not high enough in heat-resistance to serve as engineering plastics. Blending of iPP with engineering thermoplastics has been an effective way to improve the properties of iPP. ${ }^{1-4}$

Syndiotactic polystyrene (sPS) is a new semicrystalline polymer with a melting temperature of about $270^{\circ} \mathrm{C}$. Its high heat-resistance and modulus of elasticity, excellent resistance to chemicals and relatively fast crystallization rate make sPS a potential thermoplastic for a large number of applications in automotive and electronic industries. ${ }^{5}$ It has gained increasing academic and industrial interests since its first successful synthesis using a metallocene catalyst as reported by Ishihara. ${ }^{6}$ Despite these desirable properties, a disadvantage of sPS is its inherent brittleness. Thus the application of sPS might be found favorable predominantly in its blends with other polymers or com- posites. Though polymeric blends based on sPS and thermoplastic or elastomeric polymers have been reported in patent literature ${ }^{7-11}$ only a few research papers have been published. ${ }^{12-16}$ Polymers produced by blending SPS and iPP are certainly an ideal combination to create new products with balanced properties providing that the advantage of one component can compensate the deficiencies of the other. Attempts have been made to make use of sPS to modify other polymers. In this work, we use minor amounts of SPS to modify iPP, aiming at improving the heat-resistance of the latter.

It is well established that simple blends of two immiscible polymers usually have large discrete dispersed phases and weak interfacial adhesion, resulting in poor mechanical properties. A compatibilizer is usually required, which reduces the interfacial tension between the two phases, leading to a reduction in the domain size and a finer dispersion of one phase in the other, and enhances adhesion by coupling the phases together and stabilizing the dispersed phase against coalescence. ${ }^{17}$ The most desirable compatibilizer for iPP/sPS blends would be a block copolymer contain-

\footnotetext{
${ }^{\dagger}$ To whom correspondence should be addressed (E-mail: mebchen@ust.hk).

${ }^{\dagger}$ Present Address: Department of Mechanical Engineering, Hong Kong University of Science \& Technology, Clear Water Bay, Hong Kong.
} 
Compatibilization Effect of SEBS Copolymers on iPP/sPS Blend

Table I. Materials used in the present study

\begin{tabular}{|c|c|c|c|c|}
\hline Designation & Source & \multicolumn{2}{|c|}{ Molecular characteristics } & $\begin{array}{l}\text { PS } \\
(\%)\end{array}$ \\
\hline iPP (1330) & Yanshan Petrochemical Corp. & \multirow{2}{*}{\multicolumn{2}{|c|}{$\begin{array}{c}\mathrm{MI}=2.0(\mathrm{~g} / 10 \min ) \\
M_{\mathrm{w}}=25 \times 10^{4}, M_{\mathrm{n}}=9 \times 10^{4} \\
M_{\mathrm{n}}\left(\times 10^{-3}\right)\end{array}$}} & - \\
\hline \multirow[t]{2}{*}{ sPS, Questra F2250 } & \multirow[t]{2}{*}{ Dow Chemical Co. } & & & - \\
\hline & & PS block & EB block & \\
\hline Kraton $1651(\mathrm{KH})$ & Shell Chemical Co. & 29 & 116 & 32 \\
\hline Kraton $1650(\mathrm{KM})$ & Shell Chemical Co. & 10.3 & 53.3 & 29 \\
\hline Kraton 1652 (KL) & Shell Chemical Co. & 7.0 & 37.5 & 32 \\
\hline
\end{tabular}

ing sPS and iPP blocks, but such a block copolymer could hardly be realized due to limitation in polymerization mechanisms.

Block copolymers, such as poly(styrene- $b$-butadiene- $b$-styrene) (SBS) and its hydrogenated analogue (SEBS) have been demonstrated as effective compatibilizers for iPP/aPS blends. ${ }^{1,18-21}$ Studies have also shown that sPS is miscible with atactic polystyrene (aPS). ${ }^{22-25}$ Therefore, these copolymers are expected to be compatibilizers for iPP/sPS blends.

In the present study, three triblock copolymers (SEBS) with different molecular weights are chosen to compatibilize iPP/sPS blends, their effects on the morphology and mechanical properties of iPP/sPS blends being examined as a function of copolymer concentration.

The localization of a compatibilizer in a blend, depending mainly on the molecular parameters of the compatibilizer, is very important because it has much influence on the mechanical properties of the blend. TEM, though a powerful technique for examining the localization of a compatibilizer in blends containing unsaturated components ${ }^{26-28}$ is impotent for blends where all the components are saturated. In the present study, obserbvation of the distribution of compatibilizer is made on SEM examination of solvent-extracted surfaces microtomed at low temperature, whereby the effects of the distribution of compatibilizers on the mechanical properties of the blends can be evaluated. ${ }^{29}$

It is well known that polymer blends having the higher crystallization temperature component in finely dispersed droplets sometimes exhibit the retard crystallization, ${ }^{30,31}$ where the crystallization of the minor component takes place in different steps depending on undercooling conditions. This fractionated crystallization of the minor component can also occur coincidentally with the major phase in the so-called "simultaneous crystallization." ${ }^{32}$ In the present study, the effect of the compatibilizers on the melting and crystallization behavior of the blend components has also been investigated.

\section{EXPERIMENTAL}

\section{Materials}

The iPP used in this study is product from Yanshan Petrochemical Corp. (China), a sort of toughened polypropylene containing HDPE end-block, melt flow index MI $=2.0(\mathrm{~g} / \mathrm{min})$ and HDPE content is $12 \mathrm{wt} \%$. The sPS is Questra F2250 from Dow Chemical Co., $M_{\mathrm{w}}=25 \times 10^{4}, M_{\mathrm{n}}=9 \times 10^{4}(\mathrm{GPC})$. The triblock copolymers SEBS are products of the Shell Chemical Co., Kraton 1651, Kraton 1650, and Kraton 1652 (referred to as KH, KM and KL, respectively). The characteristics of these copolymers are summarized in Table I.

\section{Blends Preparation}

All the $\mathrm{iPP} / \mathrm{sPS}=80 / 20(\mathrm{w} / \mathrm{w})$ blends were prepared by melt mixing in a $60 \mathrm{~mL}$ batch Plastomill (torque rheometer, Toyo Seiki Seisaku-sho, Ltd.), at $290^{\circ} \mathrm{C}$ and 60 r.p.m. for $8 \mathrm{~min}$. The weight percentage of the block copolymer in the blends is based on the total weight of each blend (Table II). About $0.1 \%$ by weight of Inganox 1010 antioxidant was added to the blends. After mixing, the samples were compression molded into sheets with a Ya Don hydraulic press at $290^{\circ} \mathrm{C}$ and $10 \mathrm{MPa}$ for $5 \mathrm{~min}$ and cooled to room

Table II. Composition of PP/sPS* blends

\begin{tabular}{lccc}
\hline \multirow{2}{*}{$\begin{array}{l}\text { Blend } \\
\text { code }\end{array}$} & \multicolumn{3}{c}{$\begin{array}{l}\text { Compatibilizer } \\
\text { content }(w t \%)\end{array}$} \\
\cline { 2 - 4 } P-1 & KH & KM & KL \\
P-2a & 2 & - & - \\
P-4a & 4 & - & - \\
P-8a & 8 & - & - \\
P-2b & - & 2 & - \\
P-4b & - & 4 & - \\
P-8b & - & 8 & - \\
P-2c & - & - & 2 \\
P-4c & - & - & 4 \\
P-8c & - & - & 8 \\
\hline
\end{tabular}


temperature. Tensile and morphologic specimens were cut from the molded sheets.

\section{Morphological Observation}

A Hitachi S-570 scanning electron microscope was used to observe the morphology of the blends and the distribution of the compatibilizers in the blends. The cryo-fractured surfaces were made by dipping the mold-compressed samples in liquid nitrogen for more than $10 \mathrm{~min}$, and then fractured. The plane surfaces of each specimen used for observation of distribution of the compatibilizers were prepared using a LKB Ultratome microtome equipped with a glass knife. Samples were held under approximately $-100{ }^{\circ} \mathrm{C}$ under a stream of liquid nitrogen to minimize surface deformation. The samples were then explored to THF at room temperature for a moderate time to dissolve the copolymer phase at the surface of the specimens. Both the cryogenically fractured and microtomed surfaces were coated with gold prior to SEM examination.

\section{Mechanical Properties}

Tensile tests were performed at room temperature using dumbbell specimens $(20 \times 4 \times 1 \mathrm{~mm})$ on an Instron 1121 electronic testing instrument at a crosshead speed of $20 \mathrm{~mm} / \mathrm{min}$. Each tensile value reported is the average of 10 to 12 tests.

\section{Thermal Analysis}

The melting and crystallization behavior of the blend was studied using a Perkin-Elmer DSC-7 under nitrogen atmosphere. To ensure comparable thermal history, all specimens were first heated to $300^{\circ} \mathrm{C}$ for $5 \mathrm{~min}$, then cooled to $50^{\circ} \mathrm{C}$ and reheated to $300^{\circ} \mathrm{C}$, all at a rate of $10^{\circ} \mathrm{C} / \mathrm{min}$.

\section{Vicat Softening Temperature}

Vicat softening point of iPP/sPS blends were measured using a Perkin-Elmer TMA-7. The specimens (2.5 mm thick) were loaded with a static force of $100 \mathrm{mN} / \mathrm{mm}^{2}$. The temperature was raised from 0 to $200^{\circ} \mathrm{C}$ at a rate of $5^{\circ} \mathrm{C} / \mathrm{min}$ under a nitrogen atmosphere. The Vicat softening point was taken at a penetration of $1 \mathrm{~mm}$.

\section{RESULTS AND DISCUSSION}

\section{Morphology of the Blends}

The uncompatibilized iPP/sPS (80/20) blend shows the typical morphology of an incompatible blend with a very large domain size and smooth interface. The size of dispersed sPS domain ranges from ca. $1 \mu \mathrm{m}$ to $15 \mu \mathrm{m}$. Furthermore, many spherical holes are observed, due to a very poor adhesion between the two phases (Figure 1a).

Figure $1 \mathrm{~b}-\mathrm{d}$ shows the fracture surfaces of the blends to which $4 \mathrm{wt} \%$ of the three triblock copolymers with different molecular weight (MW) have been added. When the higher molecular weight triblock copolymer $\mathrm{KH}$ is used as the compatibilizer, the size of sPS domain is decreased and a fine dispersion is obtained. However, still many spherical holes are observed, denoting no very strong interfacial adhesion between the two phases (Figure 1b).

In contrast, the addition of $\mathrm{KM}$ and $\mathrm{KL}$ to the blends can cause a significant reduction in SPS domain sizes and much finer dispersion of the sPS microphases. The interface between the iPP and sPS phase become indistinguishable and it is difficult to distinguish the sPS particles from the matrix. Fracturing of all the sPS particles suggests a very strong interfacial adhesion between the two phases (Figure 1c, 1d).

Thus, it is clear that the medium and the lower MW triblock copolymer are more effective compatibilizers for iPP/sPS blend than the higher MW KH. Such a result is in accord with previously results of Taha, ${ }^{33}$ where the same three triblock copolymers (Kraton 1651,1650 and 1652) were used in compatibilizing LDPE/aPS blends. The relatively poor compatibilizing ability of the higher MW copolymer KH is due to the fact that the melt viscosity for KH is much higher than those of the two others (KM, KL) and the high melt viscosity prevents $\mathrm{KH}$ from migrating effectively into the interface. Another factor is that the higher MW copolymer tends to form micelles more readily, ${ }^{34}$ which would decrease the compatibilizing effect.

\section{Distribution of the Compatibilizers}

Figure 2 shows SEM micrographs of the solventetched microtomed surfaces of iPP/sPS blends compatibilized by varying amounts of the higher MW copolymer KH. Comparing with the SEM micrograph of the un-etched microtomed surface of the blend (Figure 3), the localization of the compatibilizer in the blends can be displayed by voids remaining after dissolving the compatibilizer phase with THF. When $2 \mathrm{wt} \% \mathrm{KH}$ had been added to the blend, part of the $\mathrm{KH}$ was found to locate at the interface between the sPS particles and the iPP matrix (shown by ring bands around sPS particles), the rest dispersed in the iPP phase as microdomains (as indicated by the tiny holes) (Figure 2a).

On addition of more $\mathrm{KH}$ to the blend $(4 \mathrm{wt} \%$ and $8 \mathrm{wt} \%$ ), the part of $\mathrm{KH}$ locating at the interface as ring bands remains with no significant change; the excess copolymer, however, tends to distribute in the iPP matrix to form micelles or micellar aggregation (Figure $2 \mathrm{~b}, 2 \mathrm{c})$. Unlike the distribution of $\mathrm{KH}$ in the blends, majority of KM in the blend concentrate at the inter- 

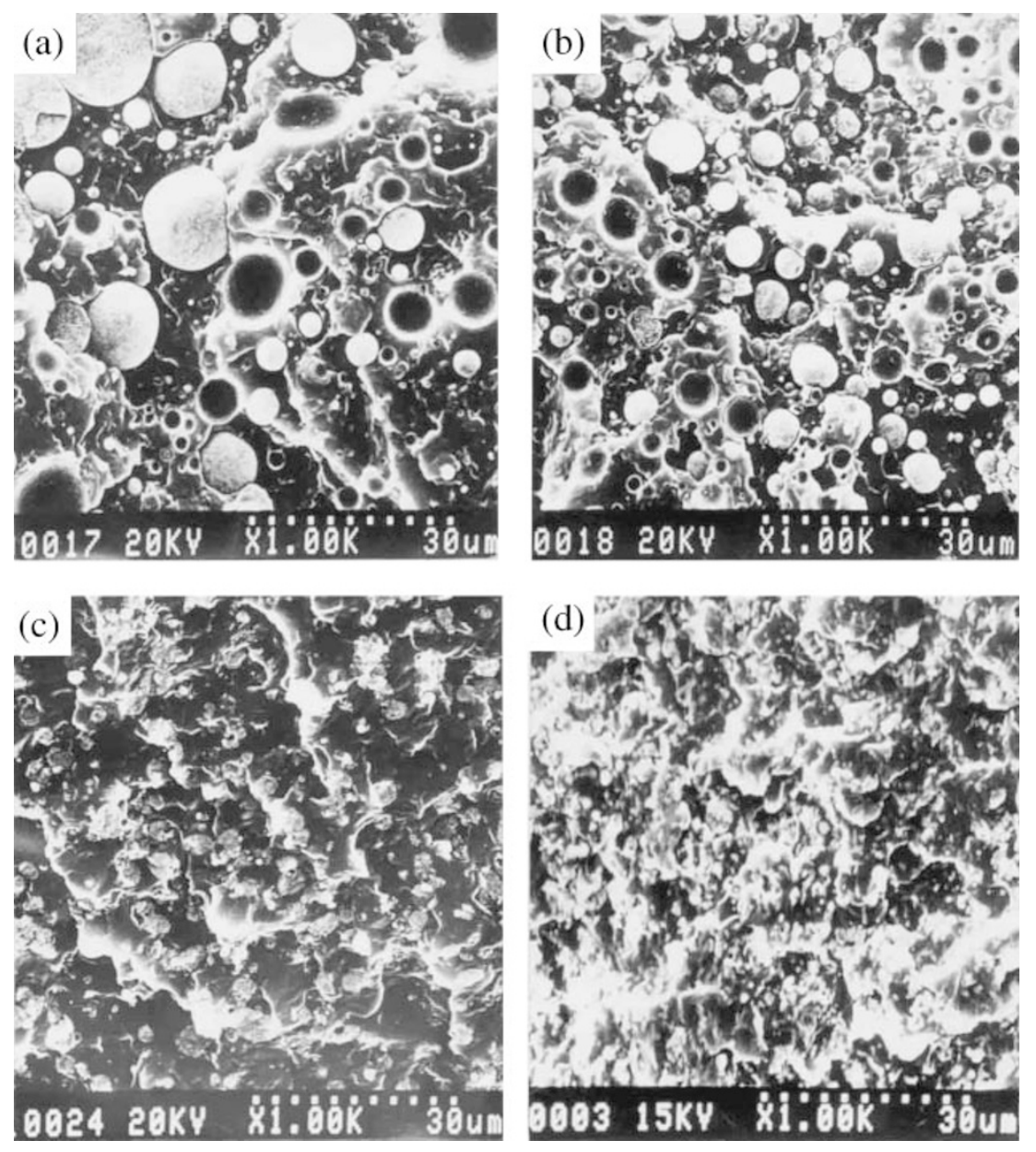

Figure 1. SEM micrographs of fractured surface of iPP/sPS (80/20) blend compatibilized with 4 wt\% of various compatibilizers: (a) None; (b) KH; (c) KM; (d) KL.

face between the two phases at either lower or higher copolymer content. Only a little was found to disperse in the iPP matrix (Figure 4). Very similar distribution of $\mathrm{KL}$ in the iPP/sPS blend was observed. It is worth noticing that no void was observed in SPS dispersed phases, indicating no compatibilizer locates in sPS phases (Figure 5).

\section{Tensile Properties of the Blends}

Figure 6 shows the tensile properties of iPP/sPS $(80 / 20)$ blends modified by varying amounts of the three SEBS copolymers. The uncompatibilized blend exhibits relatively low strength due to the very poor adhesion between sPS particles and the iPP matrix. Addition of either KM or KL to the blend results in significant improvements in the strength of the blends. When $\mathrm{KH}$ is added, however, the strength of the blends increases marginally only at a low KH content (Figure 6a). The strength of polymer blends depends, among other factors, on the morphology and on the interfacial properties. Much finer dispersion of sPS in iPP matrix and very strong interfacial adhesion is ob- tained between the two phases in both $\mathrm{KM}$ and $\mathrm{KL}$ compatibilized blends. The higher MW compatibilizer, KH, however, has little effect on tensile strength of the blends because of its relatively poor ability in compatibilizing the blends. It is also noticed that the blends with middle compatibilizer content show the maximum value of strength. This result can be explained in the light of the following facts: When blend contains moderated amount of compatibilizer, most of the compatibilizer locates at the interfacial area, which enhances the interfacial adhesion; however, when more compatibilizer is added to the blend, only part of them concentrate at the interface, the rest will distribute in the iPP matrix in the form of micro-particles. Since the strength of this rubbery block copolymer is much lower than either the PP matrix or the dispersed sPS phase, the part that distributes in iPP matrix causes a decrease in tensile strength of the blends.

The elongation at break of iPP/sPS blends as a function of the compatibilizer content is shown in Figure $6 \mathrm{~b}$. The unmodified iPP/sPS blend exhibits a very 

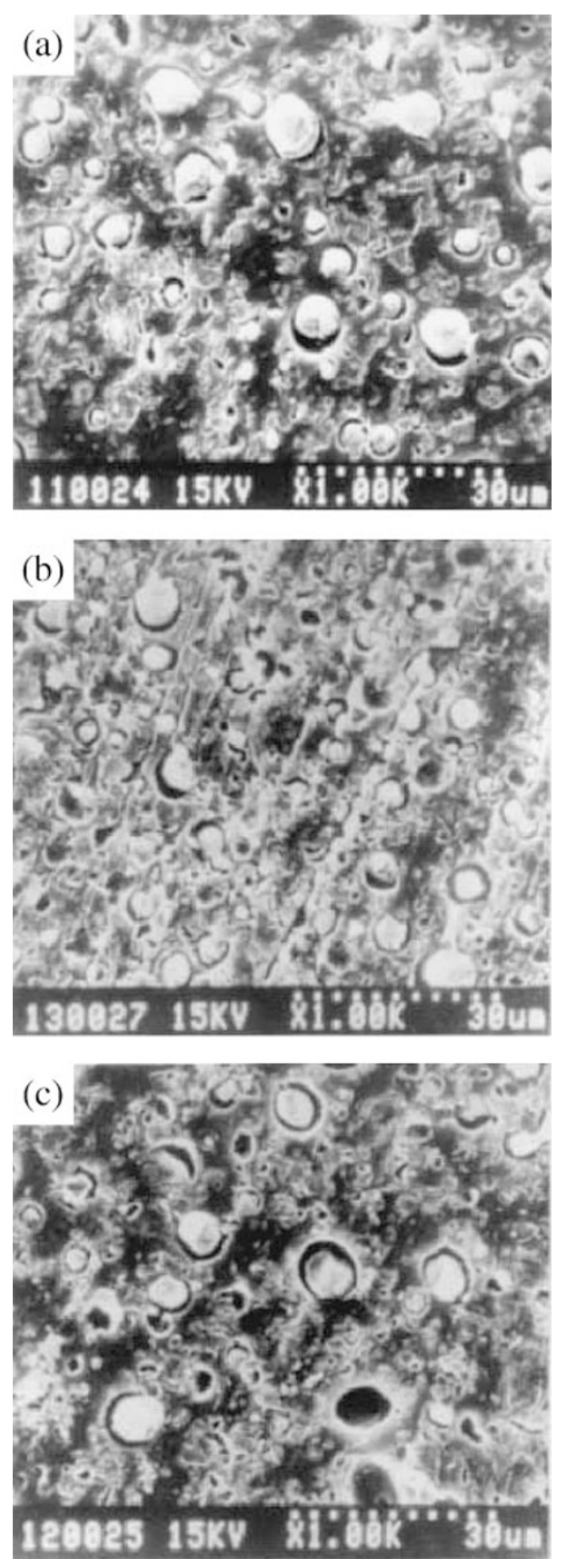

Figure 2. SEM micrographs of the solvent-etched microtomed surfaces of iPP/sPS $(80 / 20)$ blends compatibilized with various amounts of $\mathrm{KH}$ : (a) $2 \mathrm{wt} \%$; (b) $4 \mathrm{wt} \%$; (c) $8 \mathrm{wt} \%$.

low elongation at break, owing to the large size of the dispersed sPS and the very weak interfacial adhesion between the two phases. The elongation at break is significantly improved on addition of $2 \mathrm{wt} \%$ of the $\mathrm{KH}$ to the blend and increases sharply with increasing copolymer content. In contrast, the elongation at break is little affected by the addition of either KM or KL.

The high toughness, attained in KH compatibilized iPP/sPS blends, can be attributed to the distribution of the compatibilizer in the blends. It has been shown that only part of $\mathrm{KH}$ locates at the interface, the rest

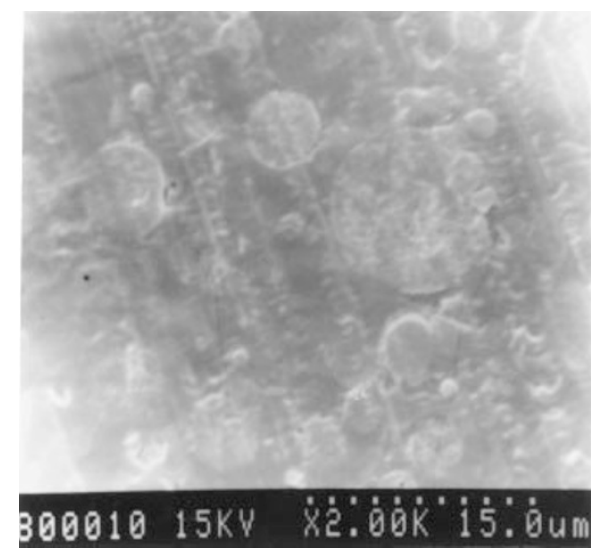

Figure 3. SEM micrograph of the unetched microtomed surface of iPP/sPS (80/20) blends compatibilized with 4\%wt KH.

dispersing in iPP phase in the form of microdomains. The compatibilizer that disperses in the iPP matrix acts as toughness modifier. In case of KM and KL, there is no such an effect since almost all the copolymers accumulate at the interfacial area. Other important factors are probably a comparatively finer dispersion of sPS particles in iPP matrix and a relatively weaker interfacial adhesion caused by KH.

Burau $^{35}$ has shown that strong adhesion between the HDPE matrix and the dispersed aPS produced by SEBS copolymer has a negative effect on the ductility of the HDPE/aPS (85/15) blend.

Figure $6 \mathrm{c}$ illustrates the effect of the quantity of the three different block copolymers on the moduli of $\mathrm{iPP} / \mathrm{sPS}$ blends. Addition of copolymers to the blends causes a decrease in modulus, decreasing with increasing copolymer content, a phenomenon also observed in other blends. ${ }^{36,37}$ The modulus of a blend depends mainly on that of the blend components besides the factor of interfacial adhesion. The copolymers used here are all elastomers whose modulus are almost two orders of magnitude lower than that of the blend components; hence, small increases in the weight fraction of this phase could significantly reduce the moduli of the blends.

Using a series of crystalline and noncrystalline SEB (SEBS) copolymers, whose EB block is either crystalline or rubbery, to modify LLDPE/aPS blends, $\mathrm{Li}^{38}$ found that the former caused an increase in modulus of the blends, whereas the latter had an opposite effect. This result has been attributed to the different interfacial structures formed by the two kinds of the copolymers. Crystalline copolymers would result in a stronger interfacial adhesion than noncrystalline copolymers. Another factor, probably more important, is that the crystalline copolymers have much higher modulus than that of the noncrystalline, leading to an increase in modulus of the blends. Heikens ${ }^{39}$ found that elastomeric block copolymer HPB- $b$-PS copoly- 

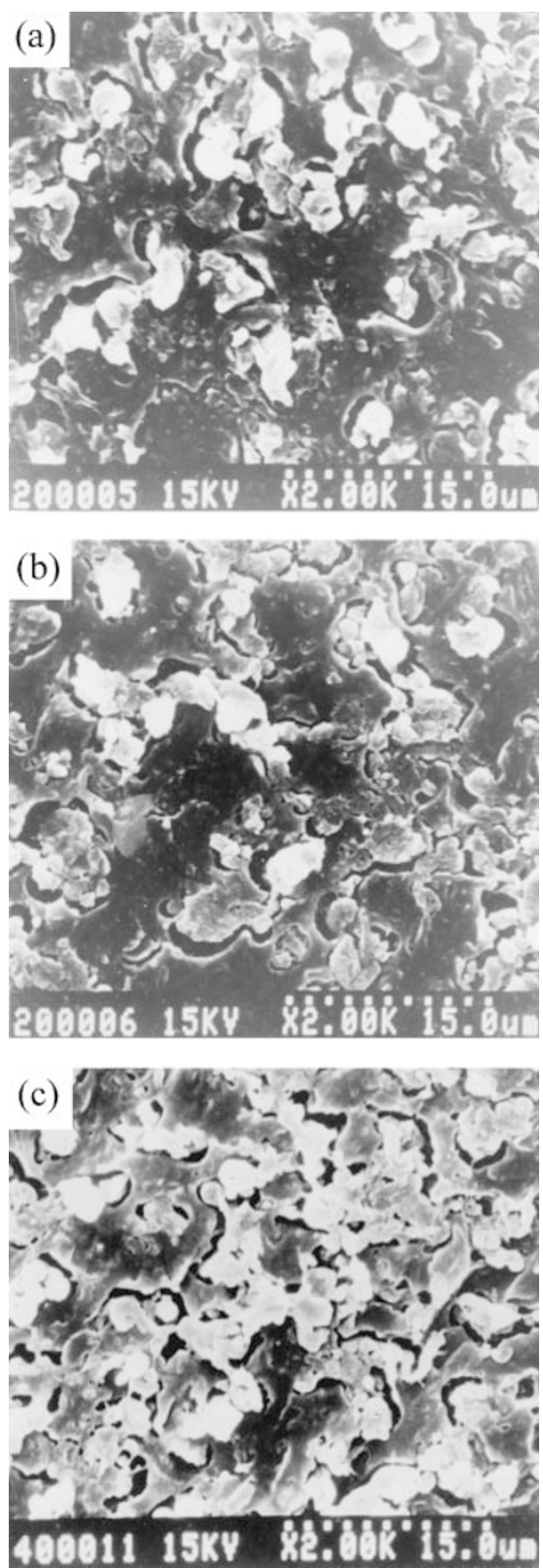

Figure 4. SEM micrographs of the solvent-etched microtomed surfaces of iPP/sPS (80/20) blends compatibilized with various amounts of KM: (a) $2 \mathrm{wt} \%$; (b) $4 \mathrm{wt} \%$; (c) $8 \mathrm{wt} \%$.

mer whose modulus is lower than the blend caused a decrease in modulus of the blend, while the graft copolymers PS- $g$-PE whose moduli correspond to that of the blend did not have this negative effect. This is another evidence to support the above explanation.

\section{Heat-resistance of the Blend}

The object of blending iPP with sPS is to upgrade the working temperature range of the blend. Vicat softening points of the iPP/sPS (80/20) blends modified by varying amounts of the copolymers are presented in Table III. The Vicat softening point of virgin iPP (PE-blocked) is only $135^{\circ} \mathrm{C}$ while that of the iPP/
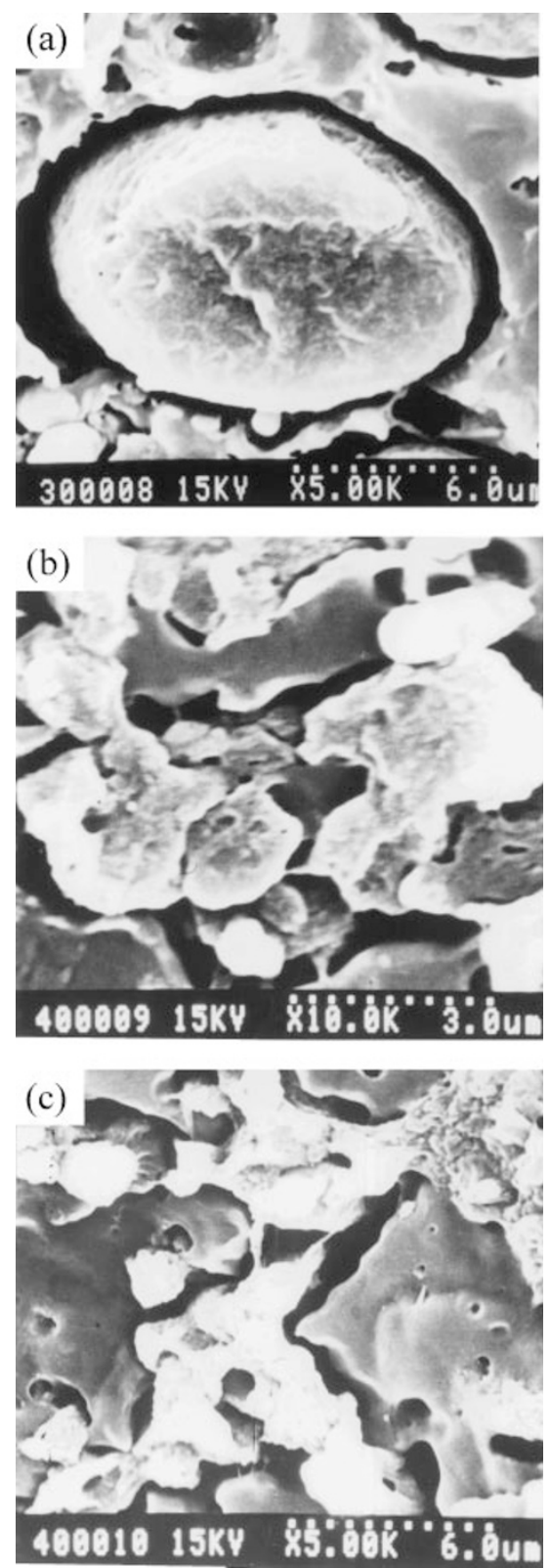

Figure 5. SEM micrographs of the solvent-etched microtomed surfaces of iPP/sPS (80/20) blends compatibilized with 4 wt\% of different compatibilizers: (a) KH; (b) KM; (c) KL (Given in details).

sPS $(80 / 20)$ blend is about $160^{\circ} \mathrm{C}$, decreasing only slightly with increasing compatibilizer content; the heat-resistance of the blend is much higher than that of the neat iPP. The value of softening temperature is dependent not only on blend composition but also on the morphology of a blend. The blends contain $4 \mathrm{wt} \% \mathrm{KM}$ and $\mathrm{KL}$ have the optimum morphology (as has been shown above), so they display relatively higher values of Vicat softening point than those of others. In general, the blends compatibilized by different copolymers exhibit roughly the same values of softening point. 


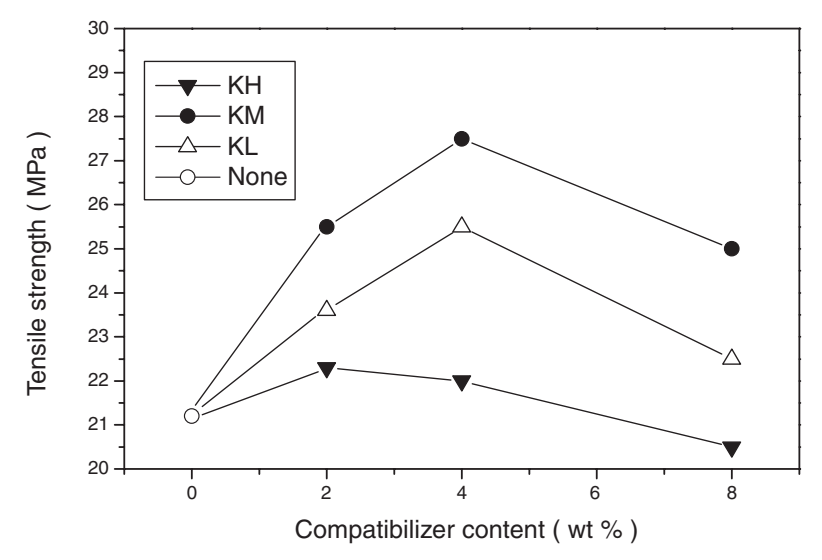

(a)

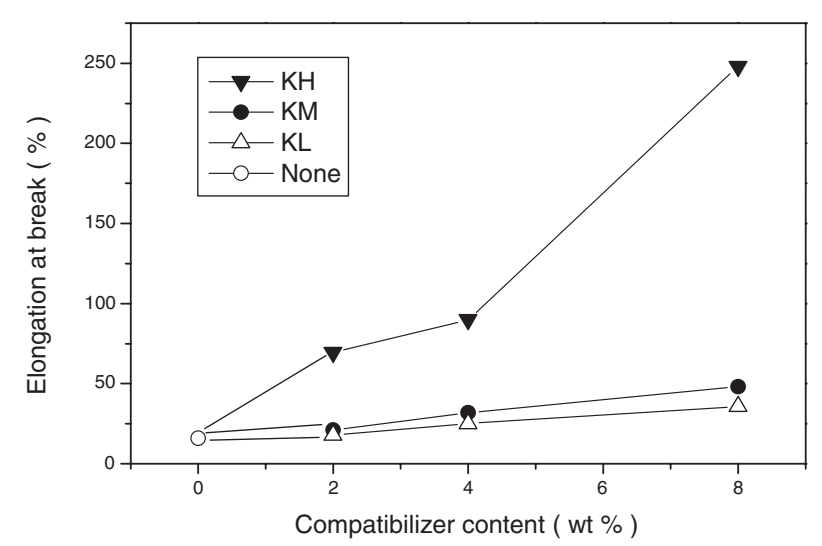

(b)

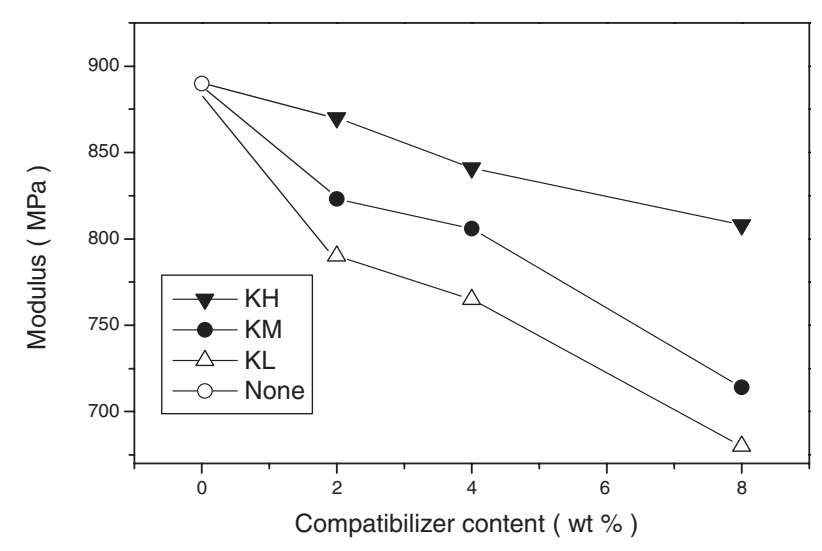

(c)

Figure 6. Effect of compatibilizers on tensile properties of iPP/sPS (80/20) blends: (a) tensile strength; (b) elongation at break; (c) modulus.

\section{Thermal Analysis}

The DSC crystallization and melting thermograms of $\mathrm{iPP} / \mathrm{sPS}$ blends compatibilized with varying amounts of $\mathrm{KH}$ and $\mathrm{KM}$ copolymers are shown in Figure 7 and Figure 8 with thermograms of neat iPP, sPS and the uncompatibilized blends as references. The iPP used in this study is virtually a copolymer containing a short HDPE end-block. Therefore, it shows two melting endothermic peaks on the heating
Table III. Vicat softening point of iPP, sPS and iPP/sPS/ SEBS* blends

\begin{tabular}{cc}
\hline Blend code & $\begin{array}{c}\text { Vicat softening } \\
\text { point }\left({ }^{\circ} \mathrm{C}\right)\end{array}$ \\
\hline iPP & 135 \\
sPS & 254 \\
P-1 & 159.5 \\
P-2a & 161 \\
P-4a & 160 \\
P-8a & 158.5 \\
P-4b & 163 \\
P-4c & 162.5 \\
\hline
\end{tabular}

${ }^{*} \mathrm{PP} / \mathrm{sPS}=80 / 20(\mathrm{w} / \mathrm{w})$

curve of the iPP. However, only one crystallization exotherm at about $112^{\circ} \mathrm{C}$ appeared on the crystallization curve of the pure iPP. This indicates that the presence of HDPE-block in the iPP can retard the crystallization of iPP, causing the two components crystallize concurrently at about the same temperature. Same phenomena was also found in PP-rich PP/HDPE blend. ${ }^{40}$

On the crystallization curves of iPP/sPS blends with and without compatibilizers, the crystallization peak at $112^{\circ} \mathrm{C}$ is split into two peaks: the large one at $115^{\circ} \mathrm{C}$ for iPP and the smaller one at $111^{\circ} \mathrm{C}$ for PE block. The presence of sPS in the blends promotes the crystallization of iPP block but has no significant effect on the $T_{\mathrm{c}}$ of PE block. The similar behavior was also observed in HDPE/sPS blends. ${ }^{12}$

It is worth noting that the crystallization peak of sPS component, which is at about $235^{\circ} \mathrm{C}$, becomes smaller on addition of compatibilizer $\mathrm{KH}$, and its intensity gradually decreasing with increments of $\mathrm{KH}$, as also reflected in the corresponding $\Delta H_{\mathrm{c}}$ values (Table IV). When the compatibilizer KM is added, the crystallization peak of sPS component disappears completely. Similar phenomenon has been observed in compatibilized PP/PA6 blends. ${ }^{41}$ It is suggested that the crystallization process of SPS separates into two discrete steps: one at $T_{\mathrm{c}}$ of itself and the other at that of iPP. With increasing the amount of compatibilizers the sPS domains becomes smaller and lost their composed character. This caused the reduction of the usual high temperature crystallization peak of sPS. The disappearance of the crystallization peak of sPS at the usual $T_{\mathrm{c}}$ suggests that almost the whole sPS crystallization took place coincidently at the usual $T_{\mathrm{c}}$ for PP, in a process of concurrent crystallization. It should be also point out that a very small part of sPS probably crystallized at its usual temperature, which could not be detected by DSC due to the low concentration of sPS in the case of KM.

The occurrence of concurrent crystallization in a 


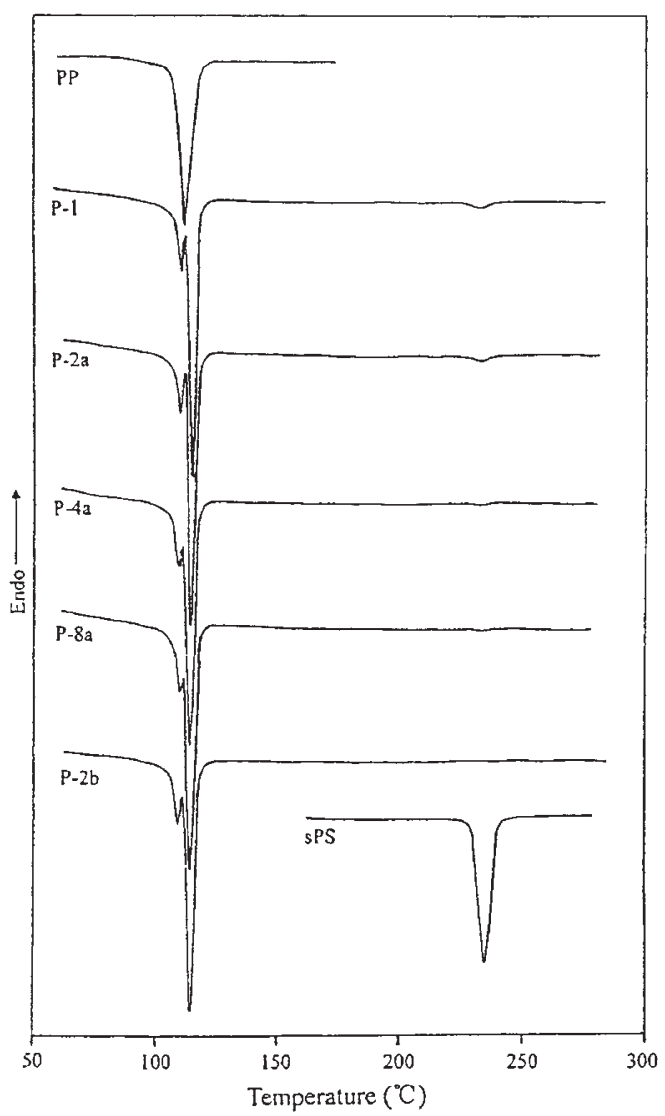

Figure 7. DSC crystallization thermograms of iPP/sPS (80/ 20) blends compatibilized with varying amounts of $\mathrm{KH}$ and KM. Cooling rate: $10^{\circ} \mathrm{C} / \mathrm{min}$.

blend has been attributed mainly to the reduction of particles sizes and a finer dispersion of the higher $T_{\mathrm{c}}$ component in the matrix. ${ }^{31}$ The number of the usually active nucleating heterogeneities is less than the number of the dispersed particles, and thus the dispersed particles in the form of supercooled molten droplets would not crystallize until the second-order nucleating impurity comes into play, or when the homogeneous nucleation begins. Such a simultaneous crystallization phenomenon has been found in blend systems like

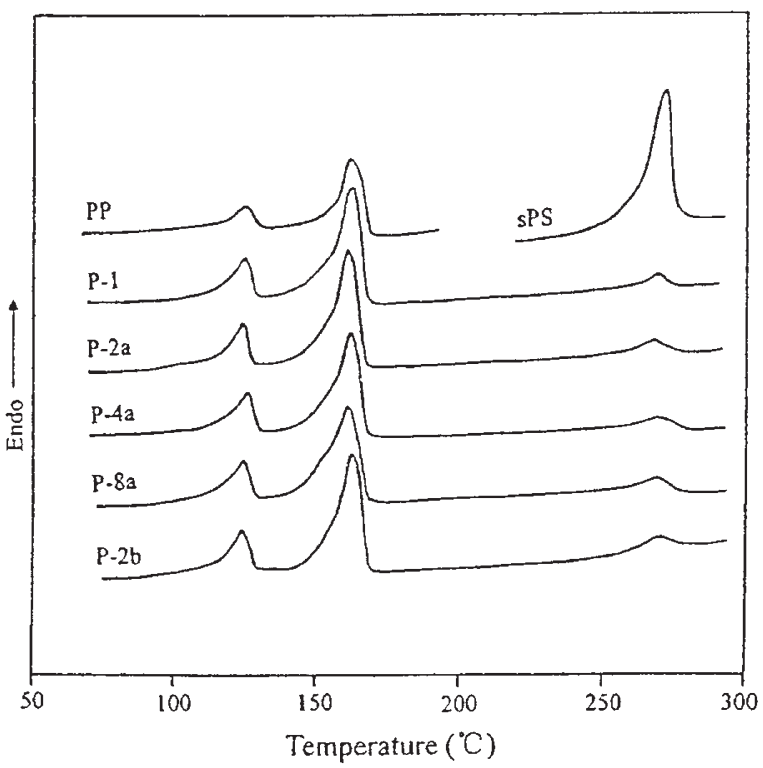

Figure 8. DSC second heating thermograms of iPP/sPS (80/ 20) blends compatibilized with varying amounts of $\mathrm{KH}$ and $\mathrm{KM}$. Heating rate: $10^{\circ} \mathrm{C} / \mathrm{min}$.

PA-6/PVDF, PBTP/PVDF, ${ }^{32}$ PP/PA- $6,{ }^{41,42}$ and PP/ PA- $12 .{ }^{43}$

Table IV presents the DSC data of non-isothermal crystallization and melting of uncompatibilized and compatibilized iPP/sPS blends as well as that of pure iPP and sPS. The melting points of iPP(PE block) stay unchanged, while those of sPS are slightly depressed by $1-2{ }^{\circ} \mathrm{C}$ compared to the plain sPS. Apparently the sPS lamellar crystallites formed in the blends are somewhat thinner than those formed in bulk. On blending, the $\Delta H_{\mathrm{f}}$ of iPP (PE block) increased significantly while that of sPS is lowered slightly. This suggests that incorporation of sPS into iPP promote crystallization of iPP (PE block). The drop in $\Delta H_{\mathrm{f}}$ (crystallinity) of sPS is obviously due to the presence of sPS as dispersed particles in iPP matrix where the crystallization ability of the former was suppressed. The normalized crystallinity of both iPP (PE block)

Table IV. Thermal characterizations of sPS, iPP and iPP/sPS blends

\begin{tabular}{|c|c|c|c|c|c|c|c|c|c|c|}
\hline \multirow{3}{*}{$\begin{array}{l}\text { Blend } \\
\text { code }\end{array}$} & \multicolumn{3}{|c|}{$T_{\mathrm{m}}\left({ }^{\circ} \mathrm{C}\right)$} & \multicolumn{3}{|c|}{$\Delta H_{\mathrm{f}}(\mathrm{J} / \mathrm{g})^{*}$} & \multicolumn{3}{|c|}{$T_{\mathrm{c}}\left({ }^{\circ} \mathrm{C}\right)$} & \multirow{3}{*}{$\begin{array}{c}\Delta H_{\mathrm{c}}(\mathrm{J} / \mathrm{g})^{*} \\
\mathrm{sPS}\end{array}$} \\
\hline & \multicolumn{2}{|c|}{$\mathrm{iPP}$} & \multirow{2}{*}{ sPS } & \multicolumn{2}{|c|}{ iPP } & \multirow{2}{*}{ sPS } & \multicolumn{2}{|c|}{ iPP } & \multirow{2}{*}{ sPS } & \\
\hline & Peak1 & Peak2 & & Peak1 & Peak2 & & Peak1 & Peak2 & & \\
\hline sPS & & & 271.3 & & & 26.9 & & & 235.1 & 24.0 \\
\hline iPP & 125.1 & 161.8 & & 17.5 & 65.6 & & 111.9 & & & \\
\hline P-1 & 125.2 & 162.1 & 269.8 & 30.4 & 70.0 & 26.1 & 111.1 & 115.8 & 233.6 & 9.1 \\
\hline $\mathrm{P}-2 \mathrm{a}$ & 124.2 & 161.1 & 268.8 & 28.4 & 68.6 & 25.4 & 110.4 & 115.7 & 234.0 & 5.1 \\
\hline $\mathrm{P}-4 \mathrm{a}$ & 125.1 & 161.8 & 270.0 & 28.3 & 65.9 & 25.1 & 110.4 & 115.2 & 234.1 & 3.9 \\
\hline P-8a & 124.3 & 160.9 & 268.4 & 27.9 & 61.9 & 23.9 & 110.4 & 114.8 & 234.6 & 2.2 \\
\hline$P-2 b$ & 124.6 & 161.8 & 269.7 & 28.1 & 68.3 & 18.5 & 109.2 & 113.8 & & \\
\hline $\mathrm{P}-4 \mathrm{~b}$ & 124.5 & 161.5 & 269.8 & 26.8 & 65.9 & 18.8 & 109.6 & 114.8 & & \\
\hline $\mathrm{P}-8 \mathrm{~b}$ & 124.0 & 161.8 & 268.9 & 24.0 & 61.7 & 17.3 & 108.5 & 114.5 & & \\
\hline
\end{tabular}

*Normalized data 
and sPS in the blend decreases on addition of increments of the compatibilizers. The decrease in crystallinity of iPP (PE block) in the compatibilized blend is probably due to the penetration of the block copolymers into the iPP phase in the interface area. The remarkably reduced size and the finer dispersion of sPS in the iPP matrix on addition of the compatibilizer retard and suppress the crystallization of sPS. Alternatively, the lowering of crystallinity of sPS may be caused by the penetration of the compatibilizer from the interface into the sPS domains.

\section{CONCLUSIONS}

The medium MW SEBS, KM, and the lower MW SEBS, KL, are efficient in reducing the sPS domain size and improving the interfacial adhesion in immiscible iPP/sPS blend, while the higher MW SEBS, KH, exhibits a relatively poor compatibilization.

$\mathrm{KH}$ locates partly at the blend interface and partly as dispersion in iPP phase in the form of microdomains. By contrast, KM accumulates mainly at the blend interface with little distribution in the iPP matrix.

Both KM and KL result in a significant improvement in tensile strength of the blends but has little effect on toughness. KH, though very effective in increasing the elongation at break, exhibits little effect on improving the strength of the blend. On addition of the compatibilizers all the blends suffer from a decrease on modulus.

Mechanical properties of iPP/sPS blends depend, besides the size of the dispersed sPS and the interfacial adhesion between the two phases, also on the compatibilizer content and its distribution in the blend.

In all these blends, iPP block crystallizes at temperature $3-4{ }^{\circ} \mathrm{C}$ higher than that of virgin $\mathrm{iPP}$, while $\mathrm{PE}$ block at its usual $T_{\mathrm{c}}$. Addition up to $8 \mathrm{wt} \%$ of $\mathrm{KH}$ causes a steady decrease in intensity of the sPS crystallization peak, while $2 \mathrm{wt} \% \mathrm{KM}$ can completely suppress the crystallization of sPS at its usual $T_{\mathrm{c}}$ and induce the almost completely concurrent crystallization of sPS and iPP at $T_{\mathrm{c}}$ of the iPP. Addition of compatibilizer to the blends causes a reduction in crystallinity of both iPP and sPS.

Acknowledgment. This research was supported by the National Natural Science Foundation of China. The authors also would like to thank Dr. James Tou for his assistance in acquiring the sPS sample from Dow Chemical Co.

\section{REFERENCES}

1. M. K. Akkapeddi and B. VanBuskirk, Adv. Polym. Technol., 11, 263 (1992).

2. Y. X. Pang, D. M. Jia, H. J. Hu, D. J. Hourston, and M. Song, Polymer, 41, 357 (2000).

3. C. Wippler, Polym. Eng. Sci., 33, 347 (1993).

4. P. T. Hielaoya, R. M. Holsti-Miettinen, J. V. Seppala, and O. T. Ikkala, J. Appl. Polym. Sci., 54, 1613 (1994).

5. R. Po and N. Cardi, Prog. Polym. Sci., 21, 47 (1996).

6. N. Ishihara, T. Seimiya, M. Kuramoto, and M. Uoi, Macromolecules, 19, 2464 (1986).

7. T. Ijitsu, T. Sumitomo, M. Sawada, and K. Funaki, Eur. Patent 318793 (July 7, 1988).

8. T. Sumitomo and K. Funaki, Eur. Patent 324398 (July 19, 1989).

9. A. Okada, Eur. Patent 587098 (Mar 16, 1994).

10. A. Okada and N. Sato, Eur. Pat. 1993, 587100 (Mar 16, 1994).

11. C. C. Chou, US. Patent 1993, 5241015 (Aug. 31, 1993).

12. B. Chen, X. L. Li, S. Xu, T. Tang, B. L. Zhou, and B. T. Huang, Polymer, 43, 953 (2002).

13. S. Xu, B. Chen, T. Tang, and B. T. Huang, Polymer, 40, 3399 (1999).

14. L. Abis, L. Abbondanza, R. Braglia, L. Castellani, G. Giannotta, and R. Po, Macromol. Chem. Phys., 201, 1732 (2000).

15. B. K. Hong and W. H. Jo, Polymer, 41, 2069 (2000).

16. B. Chen, T. Tang, S. Xu, X. Zhang, and B. T. Huang, Polym. J., 35, 141 (2003).

17. D. R. Paul and S. Newman, "Polymer Blends Vol. 2", Academic Press, New York, N.Y., 1978, Chap. 12.

18. O. O. Santana and A. Muller, Polym. Bull., 32, 471 (1994).

19. G. Radonuic, V. Musil, and I. Smit, J. Appl. Polym. Sci., 69, 2625 (1998).

20. D. Fortelny and D. Michalkova, Polym. Networks Blends, 7, 125 (1997).

21. T. Appleby, F. Cser, G. Moad, E. Rizzardo, and C. Stavropoulos, Polym. Bull., 32, 479 (1994).

22. S. Xu, B. Chen, Y. Liu, H. Chen, and B. T. Huang, Chin. Chem. Lett., 9, 407 (1998).

23. H. Ermer, R. Thomann, J. Kressler, R. Brenn, and J. Wunsch, Macromol. Chem. Phys., 198, 3639 (1997).

24. B. K. Hong, W. H. Jo, and J. Kim, Polymer, 39, 3753 (1998).

25. M. Bonnet, M. Buhk, G. Trogner, K. D. Rogausch, and J. Petermann, Acta Polym., 49, 174 (1998).

26. R. Fayt, R. Jerome, and Ph. Teyssie, J. Polym. Sci., Polym. Lett. Ed., 24, 25 (1986).

27. S. Datta and D. J. Lohse, Macromolecules, 26, 2064 (1993).

28. H. Y. Zhao and B. T. Huang, J. Polym. Sci., Polym. Phys. Ed., 36, 85 (1998).

29. F. C. Stehling, T. Huff, C. S. Speed, and J. Wissler, J. Appl. Polym. Sci., 26, 2693 (1981).

30. A. Ghijsels, N. Groesbeek, and C. W. Yip, Polymer, 23, 1913 (1982).

31. C. Robitaille and J. Prud'homme, Macromolecules, 16, 665 (1983). 
32. H. Frensch and B. J. Jungnickel, Colloid Polym. Sci., 267, 16 (1989).

33. M. Taha and V. Frerejean, J. Appl. Polym. Sci., 61, 969 (1996).

34. K. Char, H. R. Brown, and V. R. Deline, Macromolecules, 26, 4164 (1993).

35. M. N. Bureau, H. E. Kadi, J. Denault, and J. I. Dickson, Polym. Eng. Sci., 37, 377 (1997).

36. M. C. Schwarz, J. W. Barlow, and D. R. Paul, J. Appl. Polym. Sci., 37, 403 (1989).

37. S. A. Xu and C. M. Chan, Polym. J., 30, 552 (1998).

38. T. Li, V. A. Topolkareav, A. Hiltner, E. Bear, and R. P.
Quirk, J. Polym. Sci., Polym. Phys. Ed., 33, 667 (1995).

39. D. Heikens, N. Hoen, W. Barentsen, P. Peit, and H. Ladan, J. Polym. Sci., Polym. Symp., 62, 309 (1978).

40. H. P. Blom, J. W. The, T. Bremner, and A. Rudin, Polymer, 39, 4011 (1998).

41. O. T. Ikkala, R. M. Holsti-Miettinen, and J. Seppala, J. Appl. Polym. Sci., 49, 1165 (1993).

42. H. S. Moon, B. K. Ryoo, and J. K. Park, J. Polym. Sci., Polym. Phys. Ed., 32, 1427 (1994).

43. T. Tang, Z. Lei, X. Zhang, H. Chen, and B. Huang, Polymer, 36, 5061 (1995). 